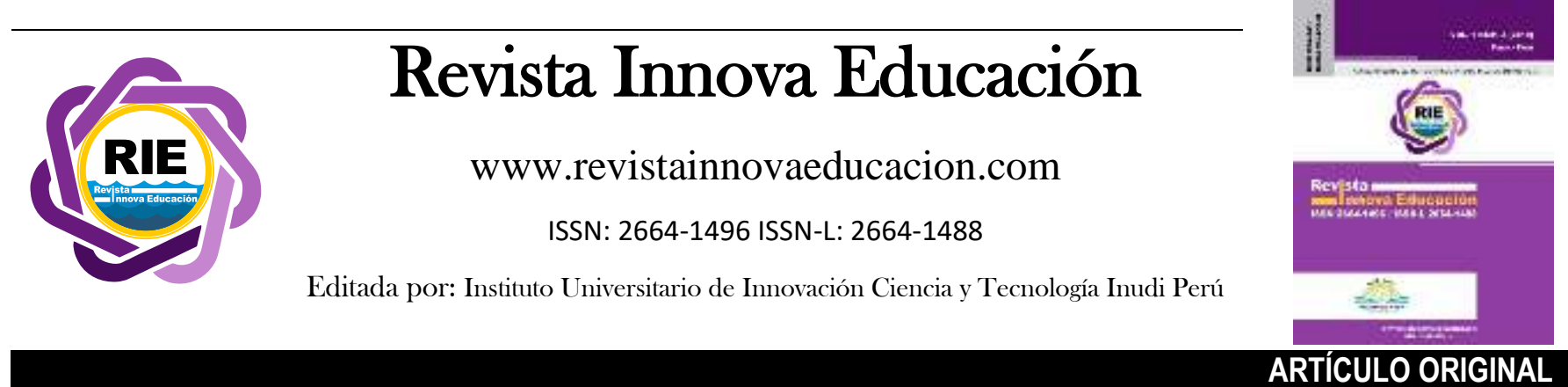

\title{
Las escuelas como espacios para la construcción de la paz y la convivencia. Una propuesta posible con niños y niñas de primarias en México
}

\author{
Schools as spaces for the construction of peace and coexistence. A possible proposal with \\ elementary school boys and girls in Mexico
}

As escolas como espaços da construção da paz e a coexistência. Uma proposta possível com crianças e meninas de eleição prévia no México

\author{
José Duarte ${ }^{1}$ \\ Cátedra CONACyT-Universidad Autónoma Metropolitana, Ciudad de México-México, México \\ (D) https://orcid.org/0000-0002-0235-1838 \\ jmduartecr@conacyt.mx (correspondencia)
}

\author{
Amys Mendoza \\ Colegio Tepeyac, San Cristóbal, Chiapas-San Cristóbal de las Casas, México \\ (iD) https://orcid.org/0000-0002-8039-5318 \\ amendoza@gmail.com
}

$\begin{array}{ll}\text { DOI (Genérico) } & : \text { https://doi.org/10.35622/j.rie.2021.01.005 } \\ \text { DOI (Documento en español) } & : \text { https://doi.org/10.35622/j.rie.2021.01.005.es } \\ \text { DOI (Document in English) } & : \text { https://doi.org/10.35622/j.rie.2021.01.005.en }\end{array}$

Recibido 30/08/2020/ Aceptado 15/01/2021 Publicado 26/01/2021

\begin{tabular}{ll}
\cline { 2 - 2 } \multicolumn{1}{c}{ ARTÍ́CULO ORIGINAL } & RESUMEN. Esta investigación tuvo como objetivo la implementación de un Proyecto \\
\cline { 2 - 3 } Prosociabilidad, & $\begin{array}{l}\text { de Educación para la Paz (PEP) en un aula de primer grado de una escuela primaria } \\
\text { ubicada en San Cristóbal de las Casas, Chiapas, México. La intervención promovió un } \\
\text { violencia escolar, } \\
\text { educación para la paz, } \\
\text { climanto de competencias prosociales en estudiantes, docentes y familias; para ello se } \\
\text { desarrollaron estrategias universales y focalizadas. Se utilizó un enfoque mixto, con } \\
\text { evaluaciones antes y después. Se recolectó información mediante la investigación- } \\
\text { acción-participativa. Algunos hallazgos indican que los PEP sistemáticos en el aula } \\
\text { disminuyen la violencia y fortalecen la prosociabilidad. Hubo una reducción en el } \\
\text { indice de violencia escolar de 1.12 a 1.10 y un aumento en la prosociabilidad estudiantil } \\
\text { de 3.34 a 3.70. Las estrategias incidieron favorablemente en el mantenimiento de un } \\
\text { clima escolar de cordialidad, mayor empatía, asertividad, mejor manejo de emociones } \\
\text { y aumento en las redes de amistad. }\end{array}$ \\
\hline
\end{tabular}

${ }^{1}$ Investigador en la Universidad Autónoma Metropolitana, México. 


\section{KEYWORDS}

Prosociability, school violence, peace education, school climate.

PALAVRAS-CHAVE
Prosociabilidade,
violência escolar,
educação para a paz,
clima escolar.

ABSTRACT. This research aimed to implement a Peace Education Project (PEP) in the first-grade classroom of a primary school located in San Cristobal de las Casas, Chiapas, Mexico. The intervention promoted a set of prosocial competencies in students, teachers, and families; for this universal and focused strategies were developed. A mixed approach was used, with evaluations before and after. Information was collected through participatory action research. Some findings indicate that systematic PEPs in the classroom decrease violence and strengthen prosociability. There was a reduction in the school violence index from 1.12 to 1.10 and an increase in student prosociability from 3.34 to 3.70 . The strategies favorably affected the maintenance of a school climate of cordiality, greater empathy, assertiveness, better management of emotions, and an increase in friendship networks.

RESUMO. Esta pesquisa teve como objetivo implementar um Projeto de Educação para a Paz (PEP) em uma sala de aula do primeiro ano de uma escola primária localizada em San Cristóbal de las Casas, Chiapas, México. A intervenção promoveu um conjunto de competências pró-sociais em alunos, professores e famílias; para isso, foram desenvolvidas estratégias universais e focadas. Foi utilizada uma abordagem mista, com avaliações antes e depois. As informações foram coletadas por meio de pesquisa-ação participativa. Algumas descobertas indicam que os PEPs sistemáticos na sala de aula diminuem a violência e fortalecem a prosociabilidade. Houve redução do índice de violência escolar de 1,12 para 1,10 e aumento da prosociabilidade dos alunos de 3,34 para 3,70. As estratégias afetaram favoravelmente a manutenção de um clima escolar de cordialidade, maior empatia, assertividade, melhor gestão das emoções e aumento das redes de amizade.

\section{INTRODUCCIÓN Y ESTADO DE CUESTIÓN}

La violencia es un problema constante en las vidas de miles de niños y niñas en el mundo (UNESCO, 2019). Este flagelo considerado una violación de derechos humanos (ONU, 2006), se manifiesta de múltiples formas: castigos físicos, ofensas, maltrato emocional, sexual, de género, entre otras (Galtung \& Dietrich, 2013), y se presentan en muchas ocasiones en entornos de protección como el hogar y la escuela (ONU, 2006; UNICEF, 2019a).

La prevención e intervención de la violencia en la infancia es un derecho fundamental garantizado por múltiples convenciones y tratados internacionales. Independientemente de las circunstancias económicas, sociales, culturales, religiosas, o étnicas, miles de niños y niñas en el mundo viven y observan situaciones reales de violencia en sus vidas (ONU, 2016; UNESCO, 2019; UNICEF, 2014, 2019a); lo que provoca daño, humillación, dolor, y desencadena secuelas que afectan su desarrollo físico, personal, social y escolar.

La diversidad de formas en que se manifiesta la violencia escolar, las múltiples consecuencias personales y sociales que conlleva en estudiantes, docentes y familias, dan cuenta que es un fenómeno complejo (Ayala, 2015), que suscita relaciones fracturadas, desconfianza, estrés, genera desgaste emocional, promueve además indisciplina, bajo aprovechamiento educativo, pérdida de respeto, poco trabajo colaborativo, dificultades en la comunicación y en la cohesión social (Chaux, 2012; Danesh, 2011; Debarbieux, 2011; OCDE, 2009; Olweus, 2006; ONU, 2016; Saucedo \& Guzmán, 2018; UNESCO, 2019).

El informe de la UNESCO, presentado en el Foro Mundial de Educación 2019 mostró datos alarmantes sobre la violencia escolar, entre ellos se estima que 246 millones de niños, niñas y 
adolescentes en el mundo son víctimas de la violencia al interior y alrededor de sus escuelas. Uno de cada tres estudiantes, es decir el $32 \%$ ha sido intimidado por sus compañeros/as en la escuela al menos una vez en el último mes. La violencia física es la más frecuente, seguida por la intimidación psicológica. El acoso afecta a niños y niñas, aunque en ellos hay una prevalencia mayor de violencia física y en las niñas es más común la psicológica. El acoso sexual es la segunda forma de violencia más común en muchas regiones del mundo. Hay un aumento en el ciberacoso por medio de dispositivos móviles. Los menores percibidos de alguna manera como "diferentes" son más propensos a sufrir intimidaciones. La apariencia física es la causa más común de intimidación, seguida por la raza, la nacionalidad y el color de la piel (ONU, 2016; UNESCO, 2019).

Este artículo tuvo como finalidad presentar los principales hallazgos de la implementación de un PEP para promover competencias prosociales y prevenir la violencia escolar en un grupo de primer grado de primaria de una institución educativa ubicada en el municipio de San Cristóbal de Las Casas, Chiapas.

A continuación, se revisan elementos teóricos y conceptuales que sustentan por un lado las consecuencias (emocionales, físicas, psicológicas, educativas, económicas y sociales) asociadas de la exposición temprana de la violencia en niños y niñas; se expone además el concepto de prosociabilidad, las competencias prosociales y cómo éstas pueden promoverse en espacios escolares. De igual forma se abordan los elementos característicos de los PEP, se identifica cómo puede construirse la paz desde lo individual, interpersonal, intergrupal, nacional o internacional.

\subsection{Consecuencias de la exposición temprana de la violencia en la infancia}

Cualquier tipo de violencia que se ejerza hacia un niño o niña tiene un efecto negativo significativo en su rendimiento y aprovechamiento académico, en su salud mental y calidad de vida; son más propensos a estar deprimidos, solitarios o ansiosos y tener baja autoestima (Debarbieux, 2011; ONU, 2016; Pinheiro, 2006; UNICEF, 2014).

El desarrollo de quienes han sido objeto de abusos graves o de abandono en la infancia, es inadecuado; tienen muchas probabilidades de tener dificultades de aprendizaje y desempeño escolar; pueden sufrir depresión, lo que puede ser motivo de la adopción de conductas de alto riesgo y comportamientos autodestructivos (UNICEF, 2014).

Muchos tienden a faltar a clases frecuentemente, son casi tres veces más propensos a sentirse como extraños en la escuela y al menos, dos veces más propensos a ausentarse de ella; obtienen resultados educativos más bajos que sus compañeros, tienen menos éxito en las pruebas de matemáticas y lectura; además, mientras más aumenta la frecuencia con que se les acosa, más disminuye su aprovechamiento académico. Por todo lo anterior, tienen mayores probabilidades de abandonar la educación formal después de culminar la educación secundaria (UNESCO, 2019).

Cuando un niño o niña crece en hogares o comunidades donde prevalece la violencia, pueden llegar a interiorizar estas conductas, naturalizarlas y adoptarlas como forma de resolver sus conflictos en la cotidianidad (Ghiso \& Ospina, 2010). Quienes padecen violencia en la primera infancia, tienen más riesgos de presentar diversas patologías en etapas posteriores de la vida (APA, 
1996; OMS, 2016; UNICEF, 2014, 2017); aumentan de manera pronunciada las probabilidades de que vuelvan a ser víctimas de la misma; en el peor de los casos, algunos investigadores como Chaux (2012), Finkelhor et al. (2009), Guerra et al. (2003), Lagerspetz et al. (1988) y Tarragona (2016) señalan que pueden llegar a repetir estas conductas hacia sus cónyuges e hijos/as. Similares consecuencias pueden sufrir los niños y niñas que presenciaron episodios violentos (Bandura et al., 1961).

Estudios longitudinales han comprobado que los niños y niñas que han sido objeto de malos tratos tienen más probabilidades de ser víctimas de violencia en las etapas posteriores de la vida y/o de convertirse en maltratadores. Maas et al. (2008) concluyeron en diversos estudios que "el tiempo, duración y cronicidad del maltrato pueden influenciar la probabilidad de que la víctima presente en el futuro comportamientos violentos"; además, que el maltrato físico en la infancia es el tipo de violencia que con mayor intensidad se asocia a conductas agresivas y violentas en la juventud, lo cual permite pensar que este tipo de fenómenos genera una transmisión transgeneracional de la violencia, y es un factor a tener en cuenta en la etiología de diversos desórdenes mentales. En ese sentido, la APA (1996) señaló que la ocurrencia de situaciones traumáticas en la niñez y la adolescencia parecen asociase a problemáticas psiquiátricas a lo largo de la vida.

La etapa más determinante en la vida de todos los niños y niñas es la de sus primeros cinco años, en ella se sientan las bases de su desarrollo físico, psicomotor y socioemocional. Está demostrada la importancia vital que tiene para el desarrollo infantil un entorno afectuoso y atento, con buena salud, alimentación equilibrada y estimulación (UNICEF, 2004). La promoción de relaciones de apego positivas con sus padres, madres, docentes y/o cuidadores ayuda a que éstos/as cultiven una autoestima equilibrada, tengan un autoconcepto positivo y autocontrol de las emociones; muchos adquieren capacidades para el control del estrés, la resolución pacífica de conflictos y para mantener relaciones armoniosas con los otros.

Un niño o niña que es querido y se le demuestra afecto de forma constante, tiene más posibilidades de llegar a ser una persona adulta feliz. Se ha comprobado que la autoconfianza, la seguridad, la capacidad de compartir y amar, e incluso las habilidades intelectuales y sociales, tienen sus raíces en las experiencias vividas durante la primera infancia en el seno familiar. En un hogar donde se respira un ambiente de cariño, de respeto, de confianza y de estabilidad, los niños o niñas se crían y se desarrollan psíquicamente más sanos y seguros, y se relacionarán con el exterior de esta misma forma, con una actitud más positiva y constructiva hacia la vida (p. 23).

Por el contrario, el desarrollo de los niños y niñas que han sido objeto de abusos graves, violencia, negligencia o abandono, es inadecuado y puede tener consecuencias trágicas en la salud mental y física que pueden perdurar toda la vida, en algunos casos, como se mencionó antes, pasar de una generación a otra (OMS, 2020; Tarragona, 2016).

Las peleas, las discusiones, los gritos y las tensiones entre los adultos son percibidas por los infantes. El miedo, la inseguridad y la tensión, que estos hechos causan, 
alteran el desarrollo psicosocial del niño o niña. Vivir en un ambiente de tensión prolongada aumenta las posibilidades de que el niño o niña se enferme y desarrolle problemas psicosomáticos como asma, trastornos del sueño y del apetito. Muchas enfermedades infantiles tienen un origen psicológico. Los menores maltratados verbal o físicamente presentan déficit en el desarrollo psicomotor, alteraciones del estado nutricional y se enferman más, son más inseguros y les cuesta más adaptarse al medio social que los rodea, ya sea en el jardín infantil, la escuela, el grupo de amigos (UNICEF, 2004, p. 51).

La violencia en la infancia trae consecuencias negativas, ya que los niños y niñas tienden a ser temerosos, dependientes, poco creativos, irritables, agresivos y mentirosos, llegan a sentir que son rechazados, desarrollar sentimientos y deseos de venganza, tener mala autoimagen (UNICEF, 2004); corren mayor peligro de incurrir en comportamientos delictivos, consumir sustancias psicotrópicas y presentar comportamientos sexuales de alto riesgo (OMS, 2020; ONU, 2016).

La exposición temprana a la violencia es crítica porque puede tener impacto en la arquitectura del cerebro en proceso de maduración, acarrear numerosas consecuencias inmediatas y permanentes para la salud física y mental. En el caso de su exposición constante, -inclusive como testigo-, la perturbación del sistema nervioso e inmunológico puede provocar limitaciones sociales, emocionales y cognitivas, así como dar lugar a comportamientos que causan enfermedades, lesiones y problemas sociales (Perry, 2001).

Los casos extremos de estrés causados por el maltrato pueden alterar el desarrollo de los sistemas nervioso e inmunológico; y contribuir a enfermedades del corazón, al cáncer, suicidio e infecciones de transmisión sexual (OMS, 2020). Para Felitti et al. (1998), la exposición a la violencia durante la niñez también puede provocar mayor predisposición a sufrir limitaciones sociales, emocionales y cognitivas durante toda la vida, a la obesidad y adoptar comportamientos de riesgo para la salud, como el uso de sustancias adictivas, tener relaciones sexuales precoces y el consumo de tabaco.

Además de las consecuencias trágicas para los individuos y familias, la violencia conlleva graves secuelas para las sociedades en todas las regiones del mundo, ocasiona graves costos económicos y sociales derivados del poco desarrollo del potencial y capacidades que tienen los menores (Pinheiro, 2006; UNICEF, 2014, 2017, 2018). El impacto inmediato y a largo plazo que tiene sobre la salud pública y los costes económicos que conlleva, socavan las inversiones que se realizan en educación, sanidad y bienestar infantil, por lo que podemos señalar que la violencia contra los niños, niñas y adolescentes, también menoscaba la capacidad productiva de las generaciones futuras (OMS, 2016; ONU, 2016).

\subsection{Prosociabilidad y conductas prosociales}

Uno de los pioneros en la investigación acerca de la prosociabilidad es el Dr. Robert Roche, quien desde 1982 define la conducta prosocial como: 
[...] aquella tendente a ayudar, beneficiar a otra persona o grupo de personas sin que exista previamente una recompensa exterior anticipada al autor. Estas acciones, a menudo, suponen algún coste, riesgo o autosacrificio por parte del autor. Y comprenden una amplia variedad de conductas: generosidad, ayuda en la pena o apuro, el compartir posesiones, la donación, la cooperación y en general la participación en actividades tendentes a mejorar el bienestar de los demás, reduciendo las injusticias sociales, las desigualdades y la violencia (p. 102).

Roche (1995) identificó una amplia gama de acciones en la interacción humana que calificarían como comportamientos prosociales, entre ellas: la ayuda y servicio físico, el cuidado, la ayuda verbal, el consuelo verbal, el dar y compartir, la confirmación y valorización positiva del otro, la escucha profunda, empatía, solidaridad, la presencia positiva y la unidad. Después de treinta y cinco años de investigación en el tema, Roche y sus colaboradores proponen modelo PROT (Teoría de Optimización Prosocial), señalando que la prosociabilidad favorece la construcción de la identidad de los individuos (optimización centrada en la persona), así como sus relaciones interpersonales y sociales (Spinrad \& Eisenberg, 2009), ello sin la búsqueda de recompensas materiales o extrínsecas.

Un segundo elemento de la prosociabilidad se asocia a la ayuda hacia los otros, generando reciprocidad positiva de calidad y solidaria, salvaguardando la identidad, autonomía, creatividad e iniciativa de los individuos o grupos implicados, pero tomando en cuenta cómo éstos quieren ser beneficiados (optimización centrada en el receptor y sus circunstancias, necesidades e identidad), (Roche \& Escotorín, 2018).

Eisenberg (1999) por su parte destaca que la prosociabilidad es la capacidad que permite distinguir entre una acción buena y una mala; entre lo que es socialmente aceptable o no. Las conductas prosociales se identifican cuando se ayuda a otras personas, lo cual implica una disposición emotiva, comportamental y cognitiva en beneficio de otros.

La definición operacional de la conducta prosocial que se utilizó en esta investigación incluye los aspectos estudiados por (Roche, 1982, 1995, 1999; Roche \& Escotorín, 2018); en primer lugar, una persona con comportamientos prosociales es alguien que ofrece ayuda a los otros, es cooperativo, solidario y altruista, expresa actitudes de proximidad, atención, escucha, empatía, disponibilidad para el servicio para con otras personas y grupos; sabe confiar, establece conversaciones que expresan acogida paciente e interesada en los contenidos y objetivos de quien habla, sabe trabajar en equipos, en entornos complejos, de incertidumbre. Es una persona responsable, creativa, acepta la diversidad, sabe solucionar conflictos sin recurrir a la agresión, todo lo anterior en pro de obtener beneficios mutuos.

Según Meece (2000), los niños y niñas prosociales suelen ser más exitosos y hacen más amigos en la escuela. Chaux (2012), Lacunza (2012), Roche y Escotorín (2018), han demostrado que promover competencias prosociales mejora el desarrollo emocional y moral de los individuos, disminuye, previene y extingue la conducta violenta, mejora las relaciones sociales y familiares; tiene un impacto positivo en la autovaloración y valoración de los otros. 
En resumen, la prosociabilidad promueve la solidaridad y la reciprocidad relacional positiva; favorece la empatía, la iniciativa y la creatividad; ayuda en la mejora del aprovechamiento educativo (Spinrad \& Eisenberg, 2009). Ayuda a optimizar los niveles de cohesión social, lo que se traduce en una mejora en la práctica de la cultura cívica, en las relaciones interpersonales (Caprara et al., 2012); además, contribuye a mantener una mejor convivencia y un mayor bienestar social y personal (Inglés et al., 2009).

\subsection{Proyectos de educación para la paz}

La UNICEF (2019) define la educación para la paz como un proceso de promoción del conocimiento, capacidades, actitudes y valores necesarios para producir cambios de comportamiento que permitan a las personas prevenir la violencia, resolver los conflictos de manera pacífica y crear condiciones que conduzcan a la paz interpersonal, intergrupal, nacional o internacional.

Galtung identifica dos tipos de paz, la negativa y positiva, la primera alude a la ausencia de guerra, de violencia y agresiones; es definida como la aparente tranquilidad que proviene del orden y la calma. La paz positiva es el proceso de realización de la justicia en los diferentes niveles de las relaciones humanas; pretende afrontar y resolver los conflictos de forma no violenta, a fin de encontrar la armonía con los otros, con la naturaleza y consigo mismo (Galtung \& Dietrich, 2013).

La filosofía de la educación para la paz sostiene que los seres humanos somos sujetos de los procesos del desarrollo biológico, emocional, intelectual, ético y espiritual (Danesh \& ClarkeHabibi, 2012). Para Galtung, un proyecto de construcción de la paz debe poner al ser humano como punto de partida, ya que las personas poseemos la capacidad para la paz (Calderón, 2009).

Cuando un ser humano está debidamente educado y capacitado, utilizará sus capacidades, talentos y energías al servicio de la creación de la paz, la justicia y la prosperidad. Los principios de una cosmovisión fundada en la paz incluyen el reconocimiento de que la humanidad es una, la unidad se expresa en la diversidad y este es su mayor reto. El reconocimiento de la diversidad no puede cumplirse por medio de la violencia, sino mediante procesos abiertos, creativos, pacíficos, justos y unificadores de toma de decisiones y de resolución de problemas (Danesh \& Clarke-Habibi, 2012, p. 308).

La implementación de PEP requiere la atención a varios elementos: a) diseño de contenidos curriculares y metodologías pedagógicas enfocadas en la construcción y mantenimiento de la paz; b) la práctica del liderazgo; c) la creación de una cultura de paz dentro y entre las escuelas; d) la generación de una atmósfera de confianza; e) la reflexión profunda y sostenida sobre las cosmovisiones personales y grupales sobre la paz y los conflictos; f) la implementación de oportunidades y actividades orientadas a la paz; g) el involucramiento de las familias y h) el mantenimiento de relaciones comunitarias cercanas (Danesh \& Clarke-Habibi, 2012).

En la actualidad las iniciativas más prometedoras para la promoción y construcción de la paz en las escuelas están a favor de estrategias multicomponentes (McMahon \& Slough, 1996), 
sistémicas y centradas en los niños y niñas; muchas de ellas se enfocan en el desarrollo y fortalecimiento de competencias para la convivencia pacífica. Utilizan varios niveles de intervención simultáneamente y abordan a la comunidad educativa en su conjunto, a través de la implementación de proyectos de educación para la paz, sesiones continuas de formación docente y familiar, desarrollo de planes de estudio con un enfoque de género y derechos humanos (Bassaleti \& González, 2017; Chaux, 2012; Danesh, 2011; McMahon \& Slough, 1996; Olweus, 2006; Roche, 1999; Roche \& Escotorín, 2018; Varela et al., 2009).

Entre los modelos y programas más efectivos se encuentra el Programa Fast Track (Conduct Problems Prevention Research Group, EE.UU.); el Modelo de Gestión de la Convivencia Escolar: Paz Educa (Chile); el Modelo de Intervenciones y Apoyo al Comportamiento Positivo (Positive Behavior Interventions and Supports del Instituto de Comportamiento Violento y Destructivo de la Universidad de Oregon, EE.UU.); el Programa Aulas en Paz (Universidad de los Andes, Colombia); el Programa Olweus, de Prevención contra el Acoso (Centro de Investigación para la Promoción de la Salud de la Universidad de Bergen, en Noruega); el Programa de Educación para la Paz de H. B. Danesh (Canadá); el Programa Nacional para la Convivencia en la Escuela (SEP-México); y el Programa Integral de la conducta prosocial (España).

Los programas multicomponentes que emplean el desarrollo de competencias prosociales para la convivencia pacífica (Roche \& Escotorín, 2018; SEP, 2017), han demostrado mayor efectividad para la construcción de la paz y la prevención de la violencia escolar. Se recomiendan intervenciones constantes, sistemáticas y duraderas para mostrar efectos significativos. El enfoque sistémico es más efectivo, ya que el impacto puede ser mayor si se interviene al individuo en su contexto y tomando en cuenta sus relaciones familiares, escolares y sociales (Compañ, 2000).

\section{MÉTODO}

El objetivo general del proyecto fue la implementación de un Proyecto de Educación para la Paz (PEP) en un aula de primer grado de una escuela primaria ubicada en el municipio San Cristóbal de las Casas, Chiapas2.

La investigación se desarrolló durante el ciclo escolar 2016-2017 y se planteó desde un enfoque mixto CUAN-CUAL (Creswell \& Plano-Clark, 2011). Para la parte cuantitativa se propuso un diseño cuasiexperimental con aplicaciones antes y después. Se aplicó un cuestionario a la docente del grupo3 y ésta administró un cuestionario que midió conductas prosociales en sus estudiantes. La etapa cualitativa se desarrolló mediante los presupuestos de la InvestigaciónAcción-Participativa (Balcázar, 2003). La maestra A participó en la elaboración e implementación de un (PEP) en su aula de primer grado durante el ciclo escolar. El objetivo de este proyecto fue la prevención de la violencia escolar y la promoción de conductas prosociales en los niños, niñas y sus familias.

\footnotetext{
${ }^{2}$ Este artículo se desprende de un proyecto más amplio titulado Modelo de prevención e intervención de la violencia escolar y familiar, fue desarrollado en once escuelas primarias de México (cuatro en Hermosillo, Sonora, durante el ciclo escolar 2014-2015) y (siete en SCLC, Chiapas durante los ciclos escolares 2016-2017 y 2017-2018). Participaron en total 3,200 estudiantes, 170 docentes, 22 directivos y 165 padres y madres de familias.

${ }^{3}$ Se referirá a esta docente con la inicial A., por respeto a su confidencialidad y anonimato.
} 
Se realizó un acompañamiento sistemático en la implementación del PEP, se utilizaron técnicas de recolección de información como observaciones de aula, entrevistas semiestructuradas con la docente del grupo, personal directivo, administrativo y grupos focales con padres y madres de familia. Las informaciones recopiladas en ambas etapas, luego de ser (tabuladas/transcritas), fueron analizadas mediante los programas SPSSS, Excel, Atlas.ti y Cmap Tools. Se aplicaron pruebas estadísticas, análisis de frecuencias y se establecieron indicadores; para la información cualitativa se utilizaron unidades hermenéuticas, se construyeron códigos y mapas de categorías que favorecieron el análisis de los discursos.

\subsection{Lugar y muestra donde se realizó la investigación}

El estado de Chiapas se ubica al sureste de la república mexicana; posee una riqueza en biodiversidad, selvas extensas que albergan una infinidad de especies animales, vegetales y minerales. Su pluralidad climática, acuífera, topográfica, cultural e histórica ofrece múltiples opciones para el cultivo de la tierra y sus recursos naturales, así como para el turismo. Históricamente es una entidad que ha experimentado problemáticas político-religiosas, luchas por la tierra, por la desigualdad social, la defensa por los usos y costumbres de sus grupos indígenas; todo ello ha tenido como resultado rezagos en el ámbito educativo, en salud, infraestructura, en el desarrollo social, así como elevados índices de marginación y pobrezas.

Las estadísticas indican que Chiapas acusa los mayores rezagos sociales y el menor grado de desarrollo económico (Villafuerte \& García, 2014); ocupa el segundo lugar en el índice de marginación más alto en el país, con un grado de rezago social muy alto (SEDESOL, 2020); la última posición del Índice de Desarrollo Humano (PNUD, 2015) y cuenta con una gran cantidad de municipios enlistados en el grupo de los 100 municipios más pobres y marginados del país.

La ciudad de SCLC está ubicada en la región Altos de Chiapas; es un lugar turístico, identificado como "Pueblo Mágico". Ha sido escenario de diversas problemáticas civiles, políticas, sociales y religiosas. Según el censo del (INEGI, 2015), habitan 209,591 personas, de ellas el $45.45 \%$ se identifica como indígena, manteniendo la composición fenotípica característica de las etnias amerindias de la región maya, son hablantes de las lenguas tsotsil y tseltal.

En este municipio se ubica la escuela primaria T4, donde se desarrolló la investigación. Esta institución tiene 75 años de ofrecer servicios de educación primaria. Es de turno matutino, actualmente trabajan 6 educadores que atienden una matrícula estudiantil aproximada de 150 estudiantes.

La muestra utilizada para la elaboración de este artículo fue el grupo de primer grado de primaria, integrado por 35 estudiantes (18 niños y 17 niñas)5; la mayoría de ellos/as tenían siete años de edad cumplidos al inicio del proyecto de investigación. La docente A. tenía cuatro años de trabajar en la institución educativa. Los niveles de participación de los padres y madres de

\footnotetext{
${ }^{4}$ Se utilizará la inicial T. para referirse al centro educativo donde se realizó la investigación.

${ }^{5}$ La decisión de trabajar con este grupo de primer grado se debió a la intención del equipo de investigación de establecer una mirada focalizada de la intervención; obtener información sobre la efectividad de las estrategias, instrumentos y metodologías; finalmente, interés de la docente del grupo, ya que ésta se encontraba realizando estudios de maestría en educación y tenía que desarrollar una intervención educativa para la obtención del grado.
} 
familia de este grupo eran altos, asistían y colaboraban en las actividades convocadas en el proyecto, estaban pendientes de las necesidades y las actividades que realizaban sus hijos e hijas. La docente señaló que mantenía una buena y directa comunicación con ellos/as.

\subsection{Componentes de la intervención}

El PEP incluyó el desarrollo de estrategias multicomponentes con estudiantes, docentes, administrativos, directivos, padres y madres de familia de la institución educativa, algunas de estas acciones eran universales y otras focalizadas (ver tabla 1).

Tabla 1: Componentes de intervención y actividades desarrolladas en el PEP.

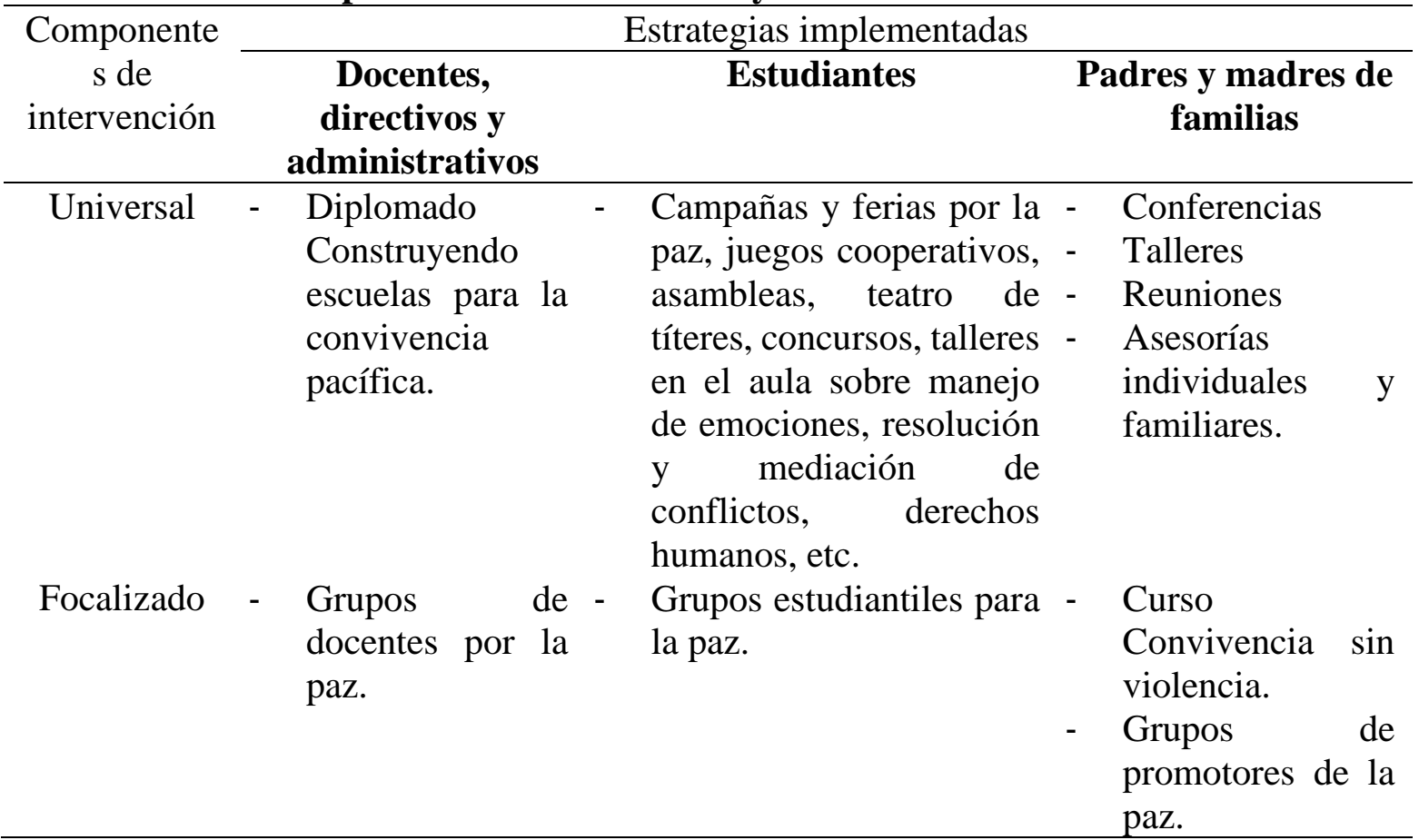

Fuente: elaboración propia.

El PEP atendió dos niveles de intervención, un componente universal ofrecido a la totalidad del colectivo estudiantil, docentes y familias en la escuela. Los profesores y administrativos participaron en diez sesiones de formación de 6.5 horas cada una a través del diplomado "Construyendo escuelas para convivir pacíficamente". El equipo de investigación implementó conferencias, talleres, pláticas y asesorías con los participantes. Estas actividades tenían la intención de prevenir la violencia y promover la convivencia pacífica.

Los docentes frente a grupo implementaron dos sesiones con sus estudiantes por semana, mediante el desarrollo de actividades lúdicas, construcción de materiales didácticos, lectura de cuentos, juegos cooperativos, campañas de prevención, dinámicas y estrategias para la resolución y mediación de conflictos pacíficamente, manejo de emociones, análisis de casos reales o ficticios, dramatizaciones, y actividades específicas donde se abordaron temas de construcción de la paz, derechos humanos, respeto y atención a la diversidad, entre otros.

El segundo nivel de intervención se desarrolló mediante el componente focalizado, para ello se conformaron grupos de docentes, de estudiantes, de padres y madres de familia. 
a) Grupo de Docentes por la Paz (GDP): se conformaron con profesores de la escuela que de manera voluntaria decidieron participar. Este colectivo tenía como objetivo el monitoreo de las actividades diseñadas en el PEP de la escuela y daban seguimiento al cronograma de actividades del proyecto.

b) Grupos Estudiantiles para la Paz (GEP): se conformaron mediante un instrumento que midió conductas prosociales en estudiantes. La información recopilada en este cuestionario ayudó a la construcción de "listas de prosociabilidad" que identificó a los estudiantes que tenían altos y bajos niveles de prosociabilidad. En cada grupo participaron seis estudiantes (cuatro con los promedios más altos y dos con el más bajo). Después de la aprobación de las familias, estos estudiantes participaron en 10-12 sesiones de formación en competencias prosociales. Estas sesiones eran desarrolladas dentro del espacio escolar y tenían una duración de una hora por semana.

c) Grupos de Promotores de la Paz (GPP): conformados por padres y madres de familia. Estas personas participaron en cinco sesiones de formación de 4 horas cada una en el curso "Convivencia sin Violencia". Se denominaron promotores de la paz y facilitaron las sesiones de los GEP apoyados por manuales diseñados para el trabajo con los estudiantes. Estos materiales contenían actividades lúdicas (juegos, dinámicas, cuentos, casos hipotéticos, materiales didácticos, dramatizaciones, cantos, etc.); incluían indicaciones para el desarrollo de las sesiones. Los padres y madres de familia participantes debían manifestar interés en ser promotores, tener disponibilidad de tiempo, ser personas dinámicas y responsables.

El PEP desarrolló, promovió y fortaleció un conjunto de competencias prosociales de cuatro tipos: emocionales (empatía, manejo de emociones); comunicativas (asertividad, argumentación y escucha activa); cognitivas (toma de perspectiva, pensamiento crítico, consideración de consecuencias, generación de opciones, interpretación de intenciones) e integradoras (resolución pacífica de conflictos, mediación, valoración de las diferencias, responsabilidad social-democrática).

\subsection{Intervención mediante el PEP con estudiantes de 1 grado}

La maestra A. participó junto a sus compañeros/as docentes y directivas de la escuela en las sesiones del diplomado. En estas sesiones de formación diseñaron el PEP, mismo que implementaron durante siete meses. El proyecto se dio inicio mediante la aplicación del instrumento que midió prosociabilidad. Este cuestionario apoyó en la identificación de las percepciones de la docente sobre la violencia, prosociabilidad, y las redes de amistad con las que contaban sus estudiantes.

El instrumento contiene 10 ítems (cinco relacionados con el nivel de prosociabilidad y cinco con el nivel de violencia). Para cuantificar las respuestas se contestaron las diferentes situaciones en las que cada estudiante podría ser prosocial o poco prosocial y se respondió: nunca, una vez, de dos a cuatro veces y cinco veces o más. En este sentido, mientras más alto el agregado de prosociabilidad, más prosocial es el estudiante y mientras más alto el agregado de violencia es 
menos prosocial. Se revisó, además, la cantidad de amigos/as que tenían los estudiantes. Para cada variable se construyó un índice individual y grupal.

El cuestionario ayudó a identificar conductas prosociales como: cantidad de amigos y amigas de cada estudiante, si se observa que los/as estudiantes ayudan a otros compañeros/as cuando están solos, si los invitan a jugar, si observan que hay alguna pelea entre sus compañeros/as, si tratan de detenerla, o avisar a su maestra/o, ayudan a algún compañero/a cuando se lastiman, también si comparten sus útiles escolares, o la comida en el momento del recreo.

De igual forma se evaluaron conductas violentas como: dar golpes, patadas o empujones a sus compañeros/as, si tratan de convencer a otros para que molesten a los demás, si tienen acciones de discriminación entre compañeros/as al no invitarlos a jugar o a participar con ellos/as en algunas actividades; si inventan chismes para reírse de algún compañero/a, si insultan o gritan ofensas o groserías dentro o fuera del aula. También fue importante conocer si los padres o madres de familia de cada estudiante asisten, participan o se involucran activamente en la formación de sus hijos/as.

Se tabuló la información obtenida en una hoja de Excel, misma que fue exportada al programa SPSSS, donde se analizaron las variables en estudio: cantidad de amigos/as, prosociabilidad y violencia escolar. Luego de hacer un análisis de medias, se estableció un índice para cada una de estas variables. Se logró obtener información sobre estas variables para identificar cuáles eran los índices obtenidos para niños y niñas. Se identificaron los estudiantes que tuvieron los índices más bajos en prosociabilidad y los más altos en violencia, con estos estudiantes la docente A. trabajó de manera más directa con actividades y técnicas diseñadas específicamente de forma personalizada.

Luego del análisis de la información estadística, se estableció la lista de prosociabilidad y se seleccionaron los estudiantes que participarían en el GEP. En el caso de 1 grado se conformaron dos grupos de seis estudiantes cada uno. La maestra A. hizo la invitación para que algunas madres y padres de familia de su grupo asistieran al curso Convivencia sin violencia. Cuatro madres participaron en el curso y fueron las facilitadoras de los dos GEP6.

Este cuestionario fue aplicado por la educadora al inicio y al finalizar el proyecto. De igual forma se utilizaron otras técnicas para recabar información; una de ellas fue la observación participativa, la misma permitió al equipo de investigación recopilar información de primera mano, de manera cercana, personal, interactiva y constante tanto del trabajo de intervención de la educadora como de la implementación del proyecto en general. Mediante un diario de campo se iban anotando sistemáticamente las informaciones acerca del desarrollo del PEP.

\section{RESULTADOS Y DISCUSIÓN}

En este apartado se presentan los cambios observados en las variables prosociabilidad, violencia escolar y redes de amistad registrados en las evaluaciones pretest y postest aplicadas a los estudiantes del primer grado de la escuela T. El diseño cuasiexperimental permitió comparar

\footnotetext{
${ }^{6}$ Se tomó la decisión que trabajaran en parejas para que se apoyaran mutuamente en el desarrollo de las sesiones con los seis estudiantes.
} 
los resultados al iniciar el proyecto y al finalizar el mismo ${ }^{7}$; algunos de ellos se presentan a continuación.

\section{a) Prosociabilidad, violencia y redes de amistad en estudiantes de primer grado de primaria}

El instrumento para medir comportamientos prosociales, violencia escolar y redes de amistad proporcionó información valiosa que de manera inicial fue analizada para el diseño del PEP, así como para el establecimiento de las listas de prosociabilidad, que apoyaron la identificación de los estudiantes que tenían altos y bajos niveles de conductas prosociales. También ofreció datos que fundamentaron el desarrollo de las actividades dentro y fuera del aula de clases. La gráfica 1 presenta información general sobre estas tres variables:

\section{Gráfica 1. Índices pretest y postest de prosociabilidad, violencia escolar y promedio de número de amigos/as de los estudiantes del I grado de la escuela T., 2016-2017*.}

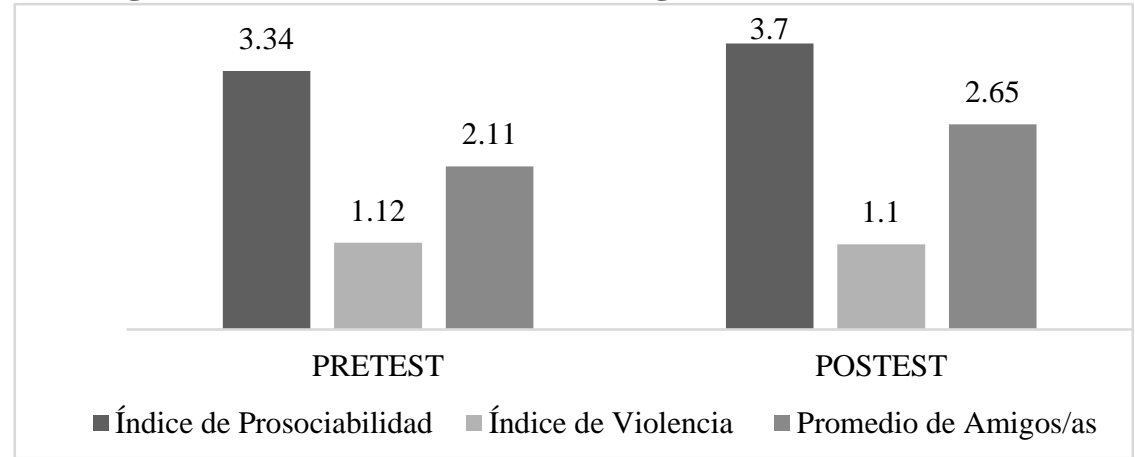

*Fuente: elaboración propia.

En la evaluación inicial el índice de prosociabilidad se estableció en 3.34, mientras que al final se observó un aumento, con un 3.70. En cuanto a la variable violencia escolar, en el pretest se estableció un índice de 1.12, mientras que en el postest hubo una disminución (1.10). En la variable número de amigos y amigas, hubo también un aumento, ya que en el inicio este promedio se estableció en un 2.11, mientras que en la evaluación final se ubicó en 2.65. Estos datos nos indican que, de forma general, la intervención fue efectiva, ya que logró aumentar el índice de prosociabilidad y el promedio general de amigos/as, mientras que se logró disminuir el índice de violencia escolar.

Estos hallazgos nos indican que la utilización de estrategias multicomponentes mediante proyectos enfocados en la construcción de la paz y la convivencia pacífica, son efectivas para la promoción de competencias prosociales y la disminución de la violencia, tal como lo han demostrado experiencias internacionales como el Programa Fast Track (McMahon \& Slough, 1996); Aulas en Paz (Chaux, 2012); Paz Educa (Varela et al., 2009); el Programa de Educación para la Paz (Danesh \& Clarke-Habibi, 2012); el Programa Integral de la conducta prosocial (Roche \& Escotorín, 2018); el Programa Olweus (Olweus, 2006); el PNCE (SEP, 2017), entre otros.

\footnotetext{
${ }^{7}$ El PEP se implementó durante siete meses.
} 
Estos programas combinan estrategias de prevención/intervención/atención primaria (universal), secundaria (focalizada) y terciarias (especializadas), es decir, utilizan un sistema continuo de prevención basado en la perspectiva de salud pública (Sugai \& Horner, 2006), y se dirigen a los estudiantes, docentes, directivos, administrativos, familias y comunidad en general, ya que consideran que la violencia escolar no es un problema aislado, sino que requiere de un abordaje integral (Pinheiro, 2006). Plantean el desarrollo y fortalecimiento de un conjunto de competencias prosociales (Caprara et al., 2012; Chaux, 2012; Roche \& Escotorín, 2018); el reforzamiento de conductas positivas; el desaliento de comportamientos inadecuados; el apoyo directo, oportuno e integral para estudiantes que requieran atención individualizada; acompañamiento en el diseño de PEP que sean inclusivos, sistemáticos, sistémicos; la promoción de climas escolares favorables; entre otras acciones.

Tabla 2. Niveles de intervención y actividades prioritarias de programas multicomponentes para la prevención/intervención de la violencia escolar*.

\begin{tabular}{|c|c|c|}
\hline Nivel primario o universal & & \\
\hline 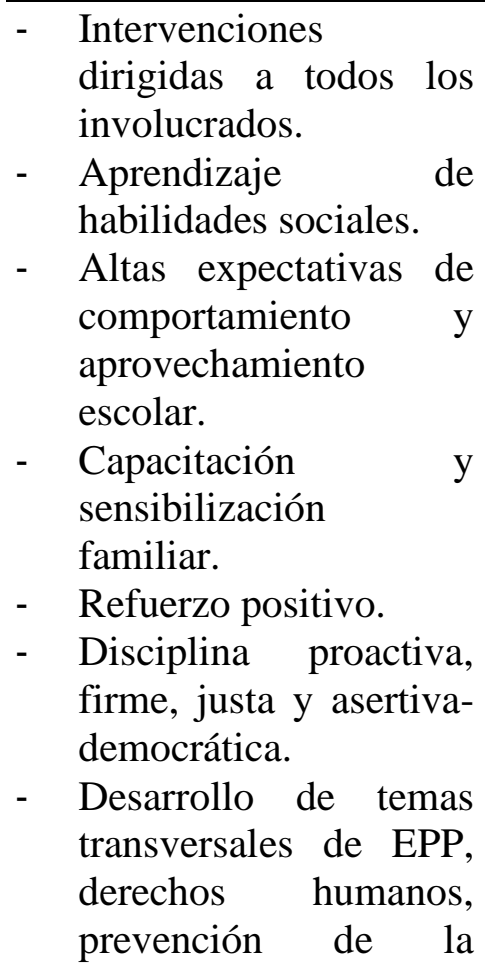 & 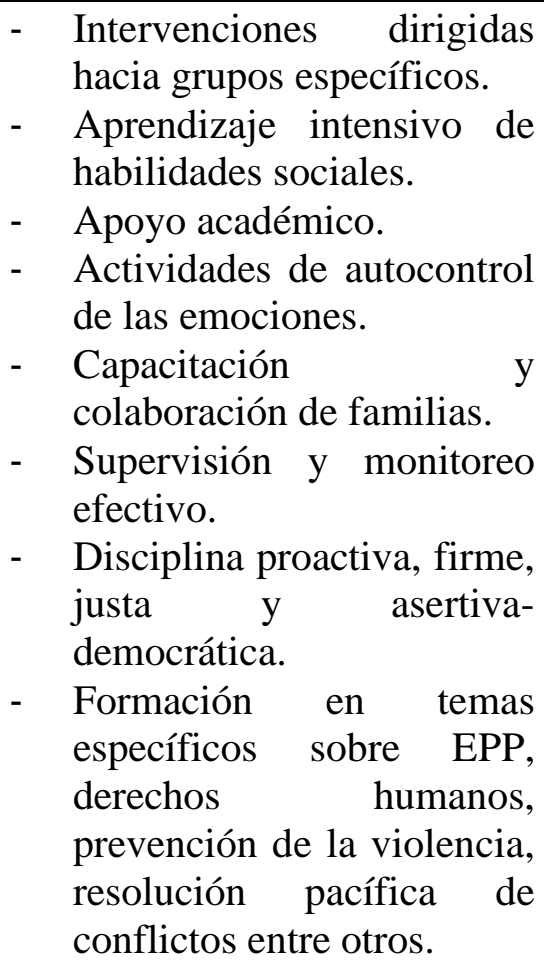 & 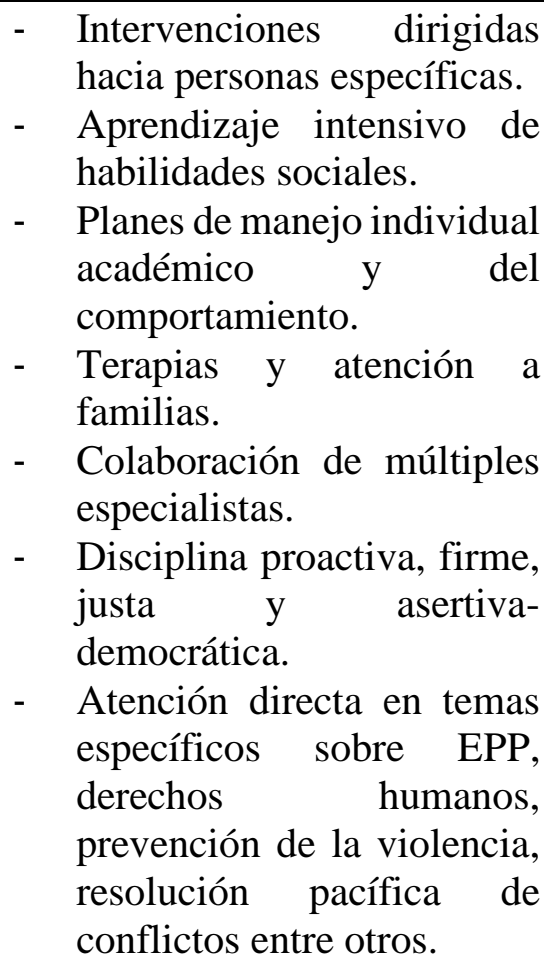 \\
\hline
\end{tabular}

violencia, resolución

pacífica de conflictos

entre otros.

Fuente: Elaboración propia con adaptaciones de las propuestas de (Chaux, 2012; Danesh \& ClarkeHabibi, 2012; McMahon \& Slough, 1996; Roche \& Escotorín, 2018; Spinrad \& Eisenberg, 2009; Sugai \& Horner, 2006; Varela et al., 2009).

Los programas más efectivos en la actualidad se enfocan en el desarrollo continuo, sistemático y transversal de estos tres niveles de prevención, intervención y atención de la violencia escolar; todos ellos se concentran en la implementación de PEP, promoviendo el fortalecimiento, desarrollo y aprendizaje de competencias prosociales para convivir pacíficamente.

La gráfica 2 presenta información comparativa del pretest y postest aplicado. Luego de un análisis de medias se pueden observar los cambios en las conductas de los estudiantes. 


\section{Gráfica 2. Índices pretest y postest obtenidos en los ítems del cuestionario para medir prosociabilidad y violencia en estudiantes del I grado de la escuela T., 2016-2017*}

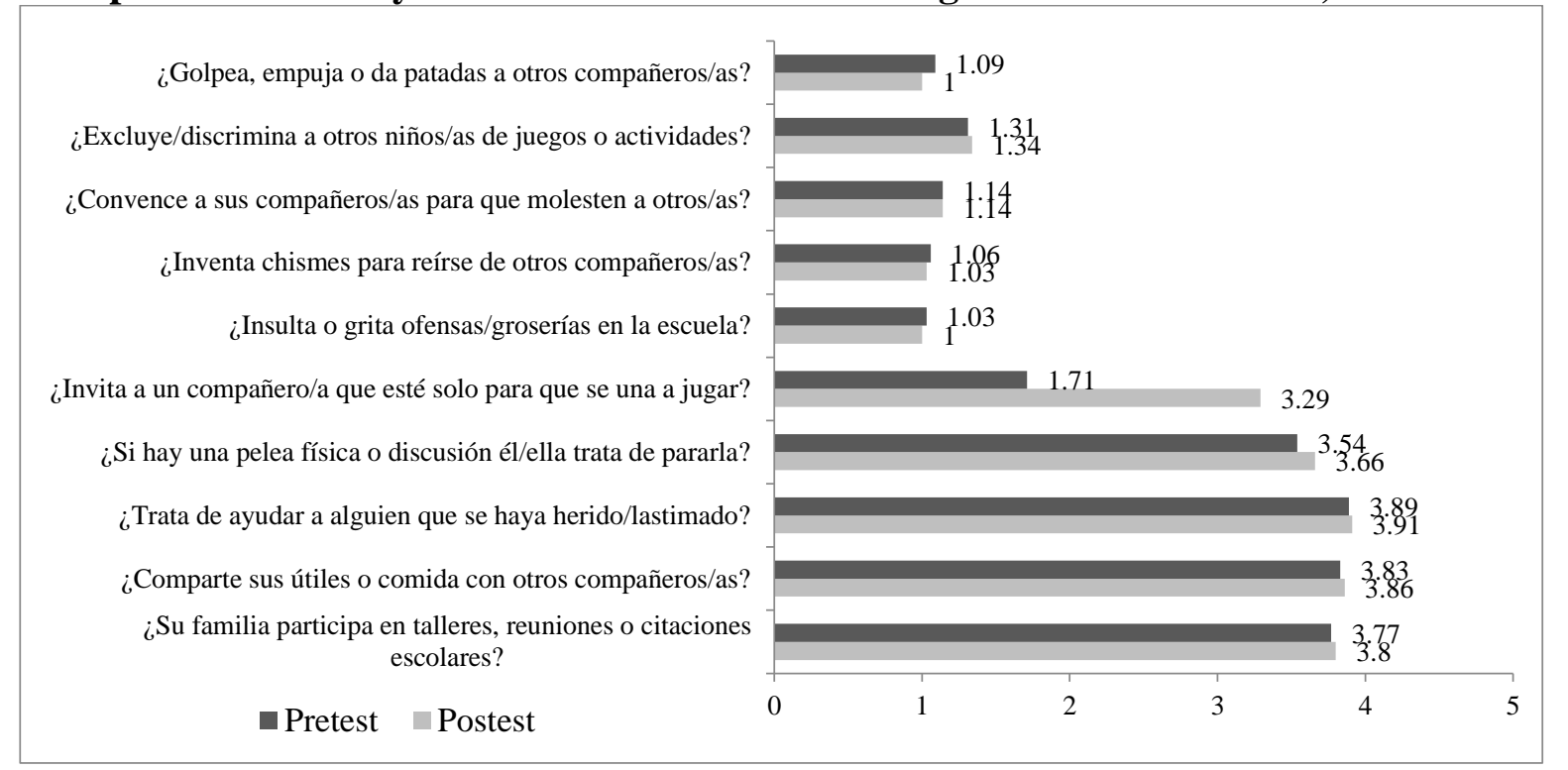

*Elaboración propia.

En las variables que miden violencia escolar hay una disminución en algunas de ellas, otras se mantienen iguales y en la variable que midió exclusión o discriminación entre compañeros en algunos juegos o actividades, hay un aumento, de 1.31 a 1.34. Esto nos indica que se elevaron los casos en que algunos niños y niñas no deseaban jugar con ciertos compañeros/as, o en algún caso tenían grupos selectivos para hacerlo.

Se puede apreciar un aumento en todas las variables que midieron conductas prosociales; aunque llama la atención la que mide las invitaciones que hacen los niños y niñas cuando alguien está solo/a. Esto nos habla de la empatía y la sensibilidad para con los otros que fue desarrollada en varias actividades mediante el trabajo en equipos, la resolución de situaciones problemáticas de forma colectiva, los juegos cooperativos, el análisis de casos hipotéticos, los cuentos analizados en clases, entre otras actividades; todo en su conjunto ayudó al desarrollo de esta competencia social.

Spinrad y Eisenberg (2009) señalan que hay estudios que han establecido relaciones entre la empatía, la agresión, los problemas de conducta y el éxito académico; además, que en el desarrollo de la empatía intervienen aspectos familiares y ambientales. Los docentes y compañeros/as en el aula pueden alentar los comportamientos prosociales, sobre todo mediante el establecimiento de relaciones cálidas, cercanas, a través del modelado y el refuerzo de las conductas positivas. Las intervenciones escolares centradas el desarrollo de habilidades sociales, el entrenamiento en empatía, el trabajo cooperativo fortalece propositivamente el comportamiento prosocial de los niños y niñas (Caprara et al., 2012; Eisenberg, 1999). 


\section{a) Acompañamiento de estudiantes identificados como "poco prosociales"}

Las listas de prosociabilidad dieron oportunidad de analizar los cambios obtenidos en los estudiantes, en particular, nos interesó conocer estos cambios en los cinco estudiantes que mantuvieron inicialmente los promedios más bajos de prosociabilidad y más altos en la variable violencia (ver gráfica 3). Con este grupo la docente del grupo programó y desarrolló actividades extras que ayudaran a reforzar las competencias prosociales.

Estos estudiantes tuvieron una intervención particular, eran observados directamente en las actividades grupales realizadas, se les motivó constantemente reforzándoles las conductas positivas o los avances que iban teniendo; se ofreció apoyo directo cuando no podían o no querían trabajar con sus compañeros; se les prestó atención y escucha cuando estaban inquietos o cuando necesitaban apoyo. Cuatro de estos estudiantes fueron incluidos también en los grupos estudiantiles para la paz, por lo que participaron en las diez sesiones de trabajo con las madres de familia promotoras de la paz. La gráfica 3 muestra las evaluaciones iniciales y finales de estos cinco estudiantes.

\section{Gráfica 3. Índices de prosociabilidad (pretest y postest) de estudiantes de 1 grado de la escuela $T$., seleccionados para dar seguimiento focalizado ${ }^{8}$, ciclo escolar 2016-2017*}

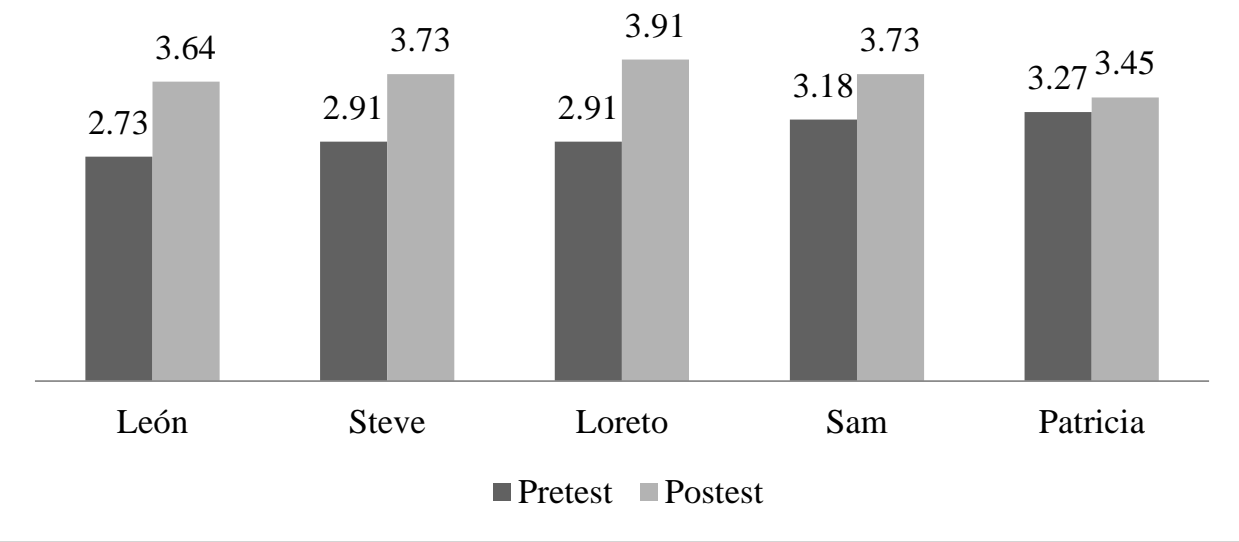

*Elaboración propia.

Al observar los índices iniciales y finales notamos grandes cambios en cada uno de ellos y ellas, se puede apreciar cómo pudieron mejorar sus conductas y caminar hacia ser cada día estudiantes más prosociales. En la entrevista realizada a la docente del grupo, ésta destacó que se trabajó de la mano con los padres y madres de familias de estos estudiantes, se les involucró para que apoyaran en el mejoramiento de las conductas de sus hijos/as y en aspectos de su aprovechamiento escolar.

El mayor avance se observa en el estudiante "Loreto", que inicialmente tenía un índice de prosociabilidad de 2.91, mientras que en la evaluación final se reflejó un índice de 3.91. Este estudiante mostraba inicialmente conductas como: gritar a sus compañeros, empujarlos dentro y fuera del salón de clases, se mostraba violento a la hora de trabajar en equipos, no participaba en clases y evidenciaba poco interés en las actividades diarias. Al finalizar el proyecto se observan

\footnotetext{
${ }^{8}$ Se colocaron pseudónimos para respetar el anonimato y la confidencialidad de estos niños/as.
} 
cambios muy notorios, la maestra A. comentó que se mostraba más tolerante, accesible, amigable, cooperativo, amistoso, participativo, y en ocasiones era líder positivo en el momento de trabajar en equipos; estos cambios fueron dados a conocer al padre y madre de familia de este estudiante.

El apoyo focalizado ofrecido a este grupo de estudiantes se basó en el programa (SWPBS9), es decir, se desarrollaron actividades sistemáticas e integradoras que tenían como objetivo promover, apoyar y fortalecer las conductas positivas de los estudiantes y no concentrarse en las negativas (Roche, 1982, 1999; Varela et al., 2009). La intervención se caracterizó por proporcionar un seguimiento continuo y cercano con ellos, apoyarlos cuando éstos/as necesitaban atención; evidenciar los logros obtenidos e incluirlos en las actividades grupales realizadas.

Realizar intervenciones focalizadas no es una tarea fácil, esto si se desea reducir el impacto, la frecuencia e intensidad de la violencia en las escuelas (Chaux, 2012; Sugai \& Horner, 2006). Los investigadores coinciden en que las estrategias sistemáticas, constantes y enfocadas en el desarrollo de competencias prosociales y -no en las situaciones de violencia, ni en abordajes punitivos-, disminuyen las probabilidades de su ocurrencia y su aumento (Danesh \& ClarkeHabibi, 2012; McMahon \& Slough, 1996; Olweus, 2006; SEP, 2017).

\section{b) Prosociabilidad y violencia escolar, diferencias en razón del sexo}

Uno de los intereses particulares en esta investigación fue conocer el aprendizaje y práctica de la prosociabilidad de niños y niñas, además, conocer las formas en que se manifiesta la violencia por grupos de sexo. La gráfica 4 ofrece información sobre los cambios en las aplicaciones (pretest y postest) por sexos en cuanto a las variables analizadas.

Gráfica 4. Índices de prosociabilidad, violencia y promedio de número de amigos/as de los estudiantes del I grado de la escuela T. por sexos, ciclo escolar 2016-2017*.

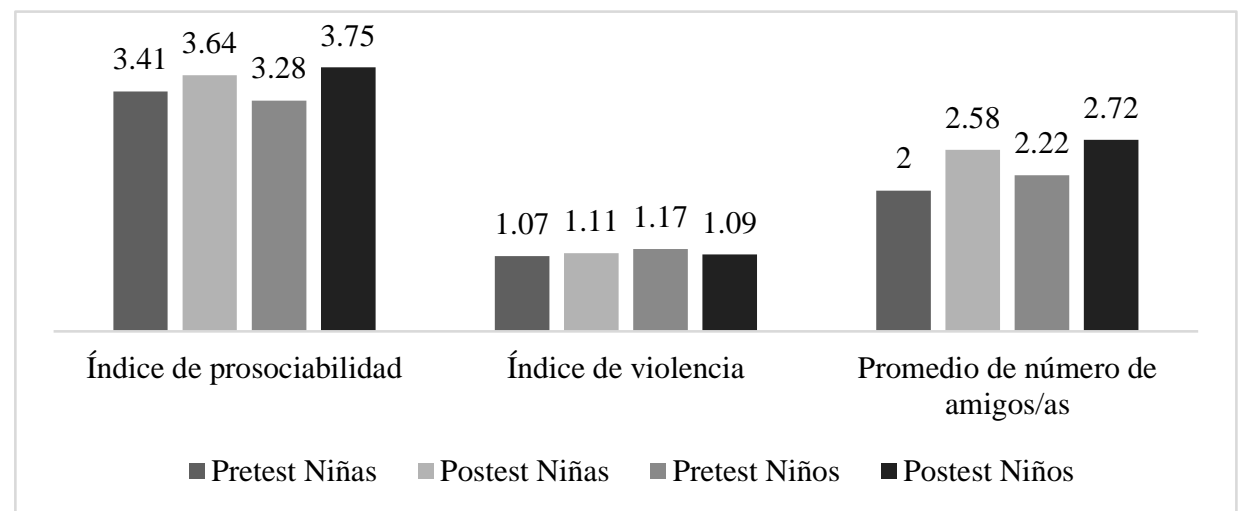

*Elaboración propia.

\footnotetext{
${ }^{9}$ Por sus siglas en inglés, es un programa de intervenciones y apoyo de comportamiento positivo escolar. Su objetivo es crear una cultura social escolar que fomente las interacciones y el comportamiento positivo, mientras desalienta los problemas de conducta. Se pretende, además, promover ambientes escolares seguros donde los estudiantes obtengan un buen aprovechamiento educativo y se construyan relaciones positivas. El enfoque se basa en enseñar a los estudiantes los comportamientos deseados, proporcionar apoyo cuando es necesario y reconocer los comportamientos esperados; todo ello basado en evidencia empírica, en la prevención y en la implementación de sistemas (Sugai \& Horner, 2006).
} 
En la gráfica 4 se observan los cambios en el índice de prosociabilidad general obtenido por niños y niñas en las evaluaciones pretest y postest. Se nota que en cuanto al índice de prosociabilidad, en ambos casos hubo aumento en las evaluaciones finales. Inicialmente las niñas obtuvieron un índice de 3.41, en el postest se observa un índice de 3.64 (hubo un aumento de 0.23 puntos porcentuales); en el caso de los niños, el índice inicial se estableció en 3.28, y el final en 3.75 (hubo un aumento de 0.47 puntos porcentuales). En las evaluaciones iniciales las niñas obtuvieron un índice mayor que los niños (0.13 puntos porcentuales), mientras que en las evaluaciones finales éstos obtuvieron un índice mayor que ellas ( 0.11 puntos porcentuales). Aunque hay diferencias porcentuales en el índice inicial y final entre niños y niñas, ellos lograron aumentar en mayor medida su índice de prosociabilidad.

En el caso del índice de violencia escolar, las niñas obtuvieron inicialmente un índice de 1.07 y en la evaluación final un índice de 1.11, es decir, hubo un aumento en este índice de 0.04 puntos porcentuales. El obtenido inicialmente por los niños fue de 1.17, mientras que en la evaluación final obtuvieron un índice de 1.09, mostrando una disminución de 0.08 puntos porcentuales. Al comparar estos resultados, se nota que en el pretest los niños obtuvieron un índice mayor (1.17) que las niñas (1.07); en el postest se refleja que ellas (1.11), obtuvieron un índice mayor que los niños (1.09).

A partir de estos resultados podemos señalar que las estrategias implementadas mediante el PEP incidieron en la disminución de la violencia identificada en los niños. En el caso de las niñas, este resultado nos proporciona información valiosa sobre las diferencias existentes en las formas en que se llevan a cabo actos de violencia en estudiantes de primer grado de primaria. Una de las limitaciones que tuvo el estudio es que la docente identificó dichas formas de violencia en los niños y las niñas de su aula de clase (antes y después de la intervención), pero no sobre los destinatarios o víctimas de estas violencias, o hacia quiénes se violenta más; lo que establece nuevas vetas para futuros proyectos.

Según la CEPAL el fenómeno de la violencia escolar se extiende a las instituciones educativas de manera generalizada y transversal; afecta a niños y niñas de todas las clases y grupos sociales; y ocurre en mayor medida en escuelas urbanas públicas (Trucco \& Inostroza, 2017). Sin embargo, las diferencias de género en las formas, intensidad y frecuencia en que se manifiesta el acoso es un debate que continúa actualmente. La investigación apunta que la violencia en el ámbito escolar se presenta más en los niños, y la forma que prevalece es la física. En el caso de las niñas, es más común la violencia psicológica y verbal (UNESCO, 2019), que incluye el esparcir rumores y el aislamiento social.

Cuando se indaga este tema en el colectivo docente, éstos refieren que los niños son más violentos que las niñas (SEP, 2009). Para Zárate y Cervantes (2011), ellos tienen una tendencia mayor a mostrar conductas violentas y menor respeto por las reglas establecidas que las niñas; además, la dirección de la violencia se encamina hacia actores del mismo sexo, lo que podría estar relacionado con la etapa del desarrollo, ya que en estas edades evitan al sexo opuesto.

Finalmente, la gráfica 4 compara el promedio en el número de amigos/as de los estudiantes, al comparar los promedios de las evaluaciones pretest y postest, hay un aumento. Se puede 
observar que para los niños el promedio inicial fue de 2.22 y para ellas fue de 2.00; el promedio final luego de la intervención de ellos fue de 2.72 y el de las niñas de 2.58. Al analizar estos datos podemos señalar que, en el caso de las niñas, tuvieron un aumento mayor que los niños. Se podría decir que el aumento en el número de amigos y amigas en ambos grupos pudo deberse al conjunto de actividades realizadas en el PEP, así como las sesiones de trabajo con los GEP que implementaron los promotores de la paz.

Estudios que analizan la relación entre el clima escolar y los niveles de aprovechamiento educativo señalan que los estudiantes que conviven en espacios de respeto, tolerancia, empatía, colaboración, trabajo en equipos, tienen una disposición mayor para aprender, que aquellos que lo hacen en ambientes violentos y hostiles (Debarbieux, 2011).

Debido al creciente auge que han tenido las investigaciones que analizan el desarrollo de la prosociabilidad en el aula, en Iberoamérica, se han empezado a desarrollar políticas educativas que integren esta dimensión como un tema central para el mejoramiento en el aprovechamiento educativo, especialmente dirigidos a los sectores más marginados. Una tendencia que se observa desde comienzos de este siglo ha sido propiciar la participación e integración de los actores involucrados en el proceso educativo: estudiantes, docentes, directivos y familias (UNESCO, 2015), es decir, invitar a toda la comunidad educativa para el desarrollo de proyectos conjuntos que tengan como objetivo promover ambientes más pacíficos e inclusivos.

La implementación de PEP con la intención de mejorar la convivencia pacífica es una tarea desafiante, por un lado, no se fomentan suficientes cursos de formación y/o actualización docente para el diseño de proyectos educativos, los programas nacionales establecen objetivos claves, pero los lineamientos y las reglas de operación son confusas y poco plausibles. Hay ambigüedades a la hora de implementar las acciones, un ejemplo es que en muchos casos se piensa que solo realizar actividades conjuntas va a propiciar el establecimiento de mejores relaciones interpersonales positivas, pero en la realidad, lo que sucede es que se constituyen grupos cerrados, acentuados por criterios de exclusión, no se evidencia un verdadero trabajo en equipos.

Las recomendaciones internacionales más efectivas para reducir la violencia escolar se enfocan en emplear estrategias no reglamentarias, más bien integradoras; una formación docente constante en temas de educación para la paz; legislación explícita y orientada en la prevención; investigaciones sólidas; planes de estudio que incluyan temas de derechos humanos y ciudadanía; así como el uso de metodologías integrales que privilegien la acción colectiva y la convivencia pacífica. Ahora bien, en la práctica cotidiana, los procesos y las relaciones interpersonales entre los diversos sistemas y actores educativos son en ocasiones difíciles de modificar por las políticas, disposiciones administrativas implementadas a través de los "reglamentos de convivencia" y por enfoques pedagógicos tradicionales que priman en el colectivo docente. Tales acciones ponen énfasis en la reducción de la violencia escolar con abordajes punitivos y de control; con estrategias basadas en amenazas y sanciones al colectivo docente y hacia los estudiantes; muchas de ellas no consideran importante la inclusión de las familias y otras instituciones sociales en los proyectos por considerarlas externas a las escuelas, todo ello reduce las oportunidades para la promoción de la convivencia. 
En este sentido, Bryk y Driscoll (1988) identificaron cinco características de la comunidad escolar que pueden estar relacionadas con la efectividad general de un centro educativo: un sistema de valores compartidos sobre el propósito, misión y visión de la escuela; una agenda común de actividades diseñada para promover interacciones sociales significativas entre los miembros de la comunidad educativa; expectativas claras para los estudiantes, el personal educativo y las familias; alto interés por el aprendizaje estudiantil; y relaciones sociales caracterizadas por el cariño y la cohesión. Podemos concluir que el PEP implementado en esta aula de clases coincide con estas cinco características y procura resaltarlas en todos los componentes de intervención.

El PEP propuesto utilizó un enfoque de formación, desarrollo y fortalecimiento de competencias prosociales para la convivencia pacífica; éstas fueron la base que orientaron las estrategias y actividades implementadas en el aula de clases y en la escuela en general. El abordaje utilizó un enfoque sistémico (Compañ, 2000), impactando el sistema áulico, docente, administrativo y familiar, así como subsistemas estructurales, dimensiones pedagógicas y académicas, dinámicas familiares, formas de comunicación, disciplina escolar y relaciones interpersonales entre los participantes. Para la ONU (2016), las intervenciones en las escuelas con un enfoque sistémico, integral y multisectorial no sólo reducen la violencia, sino que ayudan a reducir el ausentismo escolar, mejorar logros académicos, fortalecen el desarrollo de habilidades sociales y el bienestar de los niños/as.

\section{CONCLUSIONES}

Luego de la implementación del PEP, se evidencia un aumento en la práctica de competencias prosociales en los estudiantes participantes, la docente del grupo comenta que su participación en el diplomado le ayudó a mejorar prácticas que, según ella, "no eran las más apropiadas para promover la convivencia y prevenir la violencia". Las familias participaron activamente en los componentes de intervención del proyecto, asistieron a las reuniones, cuatro madres participaron en el curso "convivencia sin violencia", se realizaron cuatro talleres en el aula con una participación en promedio de 15 padres/madres. Se logró la conformación de dos GEP, esto con el apoyo de las madres promotoras de la paz.

El grupo denominado "Niños y Niñas Chiapanecos Prosociales" trabajó durante diez sesiones de una hora cada una, y el grupo "Jaguares" lo hizo en once sesiones de una hora cada una. Las promotoras de la paz de ambos grupos comentaron que la experiencia les agradó mucho, que al inicio fue difícil, ya que no se integraban todos, ni participaban mucho. Con el transcurrir de las sesiones, las actividades, juegos y dinámicas, hicieron que los niños y niñas compartieran más y aprendieran nuevas formas para resolver conflictos por medios pacíficos. Comentaron además que en poco tiempo vieron resultados en el cambio de conducta de algunos estudiantes (más receptivos, amables, tolerantes, comunicaban mejor lo que sentían y usaban en su vocabulario la palabra paz, prosocial, respeto, empatía). Señalaron que los manuales son fáciles de utilizar y apropiados para la edad de los estudiantes.

Luego de las observaciones sistemáticas realizadas en el aula de clases, se puede señalar cualitativamente que el grupo es mucho más participativo, los niños y niñas sonríen en el aula, se divierten y mantienen su atención a las indicaciones que ofrece la maestra. A la hora del recreo se 
observaron pequeños grupos que compartían su comida, otros jugaban con sus compañeros y con estudiantes de otros grados escolares. En las entrevistas realizadas con la maestra, la directora y las promotoras para la paz, indicaron varios aspectos que destacamos:

1. Hay más participación de los estudiantes en las actividades del salón, se preocupan más por realizar las actividades y se notan mejores interacciones entre ellos y ellas.

2. Los niños y niñas que antes resolvían sus conflictos mediante golpes, ahora avisan, cuestionan a los demás por las acciones agresivas que realizan, hay muy pocos eventos de peleas entre ellos y ellas.

3. Se observa la participación de las familias, están atentos, asisten a las reuniones y actividades extraescolares, colaboran, se organizan, mantienen una comunicación directa y están contentos con los resultados que se van obteniendo con sus hijos e hijas.

4. Las madres promotoras participantes comentaron que fue muy gratificante trabajar con el grupo, que aprendieron a coordinarse para las sesiones. Señalaron que valoraron mucho la confianza de los niños y niñas al contarle experiencias de sus hogares, lo que les impactó de manera personal y que en general la experiencia fue de mucho aprendizaje para ellas. Señalaron también que pudieron compartir con sus hijos/as y parejas los temas abordados en las clases.

5. Al entrevistar a la directora de la escuela, ésta refirió que nota al grupo más compenetrado, han elevado sus calificaciones y en general ha mejorado la indisciplina. Le da el crédito de los resultados positivos a las actividades realizadas por la maestra y las madres promotoras de la paz.

Algunas observaciones descritas en el diario de campo señalan que los estudiantes lograron adaptarse al grupo y pusieron en práctica una serie de competencias prosociales para convivir pacíficamente, presentaron cambios en sus conductas y se volvieron más participativos en clases, aumentaron sus redes de amistad, hubo una mejora en el clima de relaciones en la escuela, se observaron cambios en estudiantes que presentaban conductas agresivas y mayor participación de la comunidad educativa.

Al analizar la información cuantitativa obtenida en el grupo a partir del instrumento que midió la prosociabilidad, se compararon los índices iniciales y finales de los estudiantes. Los resultados indican que hubo un avance sustancial; se nota que la intervención tuvo efectos positivos en los niños y niñas participantes, por lo que podríamos concluir que la promoción de la conducta prosocial, que incluye el desarrollo de habilidades para convivir, es un elemento imprescindible si se desea mejorar el clima escolar.

Los hallazgos cualitativos y cuantitativos encontrados hacen suponer que el PEP fue efectivo en la escuela y particularmente en el aula de primer grado. La experiencia obtenida en este grupo ayudó a dar pautas específicas sobre la efectividad de los instrumentos, de los materiales y las estrategias propuestas. Al sumar todos los resultados y los avances obtenidos en los componentes de intervención, se puede concluir que la promoción de la prosociabilidad es posible, y ésta ayuda en la prevención y disminución de la violencia. 
Las escuelas debe ser el espacio ideal para la educación basada en la convivencia pacífica. Sin embargo, promover una educación integral donde los estudiantes se desarrollen tanto académica como personal y socialmente, es una tarea difícil. Desarrollar los contenidos académicos y al mismo tiempo ofrecer herramientas para convivir con los otros de manera armónica es un gran reto. En este sentido, las escuelas tienen la responsabilidad ética de ofrecer espacios que promuevan competencias prosociales para la convivencia pacífica que ayuden a formar a ciudadanos y ciudadanas íntegras que puedan hacer frente a las adversidades que convoca la vida actual.

\section{Agradecimientos / Acknowledgments:}

Expresamos el agradecimiento a los docentes del Departamento de educación de la Facultad de Educación de la Universidad Autónoma Metropolitana, México.

Conflicto de intereses / Competing interests:

Los autores declaran que no incurren en conflictos de intereses.

Rol de los autores / Authors Roles:

José Duarte: conceptualización, curación de datos, análisis formal, adquisición de fondos, investigación, metodología, administración del proyecto, recursos, software, supervisión, validación, visualización, escritura - preparación del borrador original, escritura - revisar \& amp; edición.

Amys Mendoza: conceptualización, investigación, metodología, administración del proyecto, recursos, software, supervisión, validación, visualización, escritura - preparación del borrador original, escritura - revisar \& amp; edición.

Fuentes de financiamiento / Funding:

Los autores declaran que no recibieron un fondo específico para esta investigación.

Aspectos éticos / legales; Ethics / legals:

Los autores declaran no haber incurrido en aspectos antiéticos, ni haber omitido aspectos legales en la realización de la investigación.

\section{REFERENCIAS BIBLIOGRÁFICAS}

APA. (1996). Violence and the family: Report of the American Psychological Association Presidential Task force on violence and the family. http://www.nnflp.org/apa/intro.html

Ayala, M. (2015). Violencia escolar: un problema complejo. $R a$ Xinhai, 11(4), 493-510. https://doi.org/https://doi.org/10.35197/rx.11.01.e2.2015.36.ma

Balcázar, F. (2003). Investigación acción participativa (IAP): aspectos conceptuales y dificultades de implementación. Fundamentos En Humanidades, 4(7-8), 59-77. https://www.redalyc.org/pdf/184/18400804.pdf

Bandura, A., Ross, D., \& Ross, S. (1961). Transmission of aggression through imitation of aggressive models. Journal of Abnormal and Social Psychology, 63(3), 575-582. https://pdfs.semanticscholar.org/3706/7acd33ad2ba2ed384baada06e7d74b800399.pdf

Bassaleti, R., \& González, P. (2017). Modelo de gestión de la convivencia escolar. Comunicaciones Fundación Paz Ciudadana. https://pazeduca.cl/wpcontent/themes/pazeduca/files/Modelo_Paz_Educa_2017_web.pdf

Bryk, A., \& Driscoll, M. (1988). The high school as community: Contextual influences and consequences for students and teachers. National Center on Effective Secondary Schools. https://files.eric.ed.gov/fulltext/ED302539.pdf 
Calderón, P. (2009). Teoría de conflictos de Johan Galtung. Revista Paz y Conflictos, 2, 60-81. https://www.redalyc.org/articulo.oa?id=205016389005

Caprara, G., Alessandri, G., \& Eisenberg, N. (2012). Prosociality: The contribution of traits, values, and self-efficacy beliefs. Journal of Personality and Social Psychology, 102(6), 1289-1303. https://doi.org/https://doi.org/10.1037/a0025626

Chaux, E. (2012). Educación, agresión y convivencia escolar. Taurus Santillana.

Compañ, E. (2000). El Modelo Sistémico aplicado al campo educativo. Aplicaciones, 12. http://www.iaf-alicante.es/imgs/ckfinder/files/PUB_Modelo_sistémcio_ES.pdf

Creswell, J., \& Plano-Clark, V. (2011). Designing and conducting mixed methods research (2nd ed.). Sage Publications.

Danesh, H. (2011). Education for peace. Reader. International Education for Peace Institute. http://efpinternational.org/wp-content/uploads/2011/11/efp_reader.pdf

Danesh, H., \& Clarke-Habibi, S. (2012). Manual curricular de educación para la paz. Una guía conceptual y práctica. International Education for Peace Institute.

Debarbieux, E. (2011). Refuser l'oppression quotidienne: la prévention du harcèlement à l'École. Rapport au ministre de l'éducation nationale de la jeunesse et de la vie associative. Observatoire International de la Violence à l'École. http://cache.media.education.gouv.fr/file/2011/64/5/Refuser-l-oppression-quotidienne-laprevention-du-harcelement-al-ecole_174645.pdf

Eisenberg, N. (1999). Infancia y conductas de ayuda. Morata.

Felitti, V., Anda, R., Nordenberg, D., Williamson, D., Spitz, A., Edwards, V., Koss, M., \& Marks, J. (1998). Relationship of childhood abuse and household dysfunction to many of the leading causes of death in adults. The adverse childhood experiences (ACE) study. American Journal of Preventive Medicine, 14(4), 245-258. https://doi.org/10.1016/s07493797(98)00017-

Finkelhor, D., Turner, H., Ormrod, R., Hamby, S., \& Kracke, K. (2009). Children's exposure to violence: A comprehensive national survey. Juvenile Justice Clearinghouse, 12. https://www.ncjrs.gov/pdffiles1/ojjdp/227744.pdf

Galtung, J., \& Dietrich, F. (2013). Johan Galtung: Pioneer of Peace Research. Springer.

Ghiso, A., \& Ospina, V. (2010). Naturalización de la intimidación entre escolares: un modo de construir lo social. Revista Latinoamericana de Ciencias Sociales, Niñez y Juventud, 8(1), 535-556. http://www.scielo.org.co/scielo.php?pid=S1692715X2010000100025\&script=sci_abstract\&tlng=es

Guerra, N., Huesmann, L., \& Spindler, A. (2003). Community violence exposure, social cognition, and aggression among urban elementary-school children. Child Development, 74(5), 15611576. https://doi.org/10.1111/1467-8624.00623

INEGI. (2015). Panorama sociodemográfico de Chiapas 2015. Instituto Nacional de Estadística 
https://www.inegi.org.mx/contenido/productos/prod_serv/contenidos/espanol/bvinegi/pro ductos/nueva_estruc/inter_censal/panorama/702825082154.pdf

Inglés, C., Benavides, G., Redondo, J., García-Fernández, J., Ruiz-Esteban, C., Estévez, C., \& Huescar, E. (2009). Conducta prosocial y rendimiento académico en estudiantes españoles de Educación Secundaria Obligatoria. Anales de Psicologia, 25(1), 93-101. https://www.redalyc.org/pdf/167/16711594011.pdf

Lacunza, A. (2012). Las habilidades sociales y el comportamiento prosocial. Revista Pequén Escuela de Psicología, 2(1), 1-20.

Lagerspetz, K., Björkqvist, K., \& Peltonen, T. (1988). Is indirect aggression typical of females? gender differences in aggressiveness in 11- to 12-year-old children. Aggressive Behavior, 14(6), 403-414. https://doi.org/10.1002/1098-2337(1988)14:6<403::AID$\mathrm{AB} 2480140602>3.0 . \mathrm{CO} ; 2-\mathrm{D}$

Maas, C., Herrenkohl, T., \& Sousa, C. (2008). Review of research on child maltreatment and violence in youth. Trauma, Violence \& Abuse, 9(1), 56-67. https://doi.org/10.1177/1524838007311105

McMahon, R., \& Slough, N. (1996). Family-based intervention in the Fast-Track Program. In R. Peters \& R. McMahon (Eds.), Preventing childhood disorders, substance abuse, and delinquency. (p. 400). Sage Publications.

Meece, J. (2000). Desarrollo del niño y del adolescente: compendio para educadores. Mc Graw Hill. http://www.sigeyucatan.gob.mx/materiales/1/d1/p1/4. JUDITH MEECE. Desarrollo del nino.pdf

OCDE. (2009). Creating effective teaching and learning environments: First results from TALIS. https://doi.org/978-92-64-05605-3

Olweus, D. (2006). Conductas de acoso y amenaza entre escolares (2nd ed.). Morata.

OMS. (2016). Siete estrategias para poner fin a la violencia contra los niños. https://www.who.int/violence_injury_prevention/violence/inspire/INSPIRE_ExecutiveSu mmary_ES.pdf

OMS. (2020). Violencia contra los niños. https://www.who.int/es/news-room/factsheets/detail/violence-against-children

ONU. (2006). La violencia contra niños, niñas y adolescentes. Informe de América Latina en el marco del estudio mundial de las Naciones Unidas. https://www.unicef.org/Estudio_violencia_contra.pdf

ONU. (2016). Informe anual del representante especial del Secretario General sobre la violencia contra los niños. https://www.acnur.org/fileadmin/Documentos/BDL/2016/10352.pdf

Perry, B. (2001). The neurodevelopmental impact of violence in childhood. In D. Schetky \& E. P. Benedek (Eds.), Textbook of child and adolescent forensic psychiatry (pp. 221-238). American Psychiatric Press, Inc.

Pinheiro, P. (2006). Informe mundial sobre la violencia contra los niños y niñas. ONU. 
http://www.observatoriodelainfancia.mscbs.gob.es/productos/pdf/informeMundialSobreV iolencia.pdf

PNUD. (2015). Índice de desarrollo humano en México para las entidades federativas. Programa de las Naciones Unidas para el Desarrollo. https://www.mx.undp.org/content/mexico/es/home/library/poverty/indice-de-desarrollohumano-para-las-entidades-federativas--mexi.html

Roche, R. (1982). Los origenes de la conducta altruista en ninos. Aspectos educativos y television en familia. Infancia y Aprendizaje, 5(19-20), 101-114. https://doi.org/10.1080/02103702.1982.10821948

Roche, R. (1995). Psicología y Educación para la prosocialidad. Universidad Autónoma de Barcelona.

Roche, R. (1999). Desarrollo de la inteligencia emocional y social desde los valores y actitudes prosociales en la escuela. Ciudad Nueva.

Roche, R., \& Escotorín, P. (2018). El model PROT i la responsabilitat social universitaria: Prosocialitat i transferéncia del coneixement a la práctica. Anuari de Psicología de La Societat Valenciana de Psicologia, 19(2), 109-132. https://dialnet.unirioja.es/servlet/articulo?codigo $=6864809$

Saucedo, C., \& Guzmán, C. (2018). La investigación sobre la violencia escolar en México: tendencias, tensiones y desafíos. Revista Cultura y Representaciones Sociales, 12(24), 213-245. https://doi.org/10.28965/2018-024-08

SEDESOL. (2020). Catálogo de localidades. Resumen estatal de Chiapas. http://www.microrregiones.gob.mx/catloc/Default.aspx?tipo=clave\&campo=mun\&valor= 07

SEP. (2009). Informe Nacional sobre Violencia de Género en la Educación Básica en México (E. A. Garrido (ed.); 1st ed.). http://aularedim.net/wp-content/uploads/violencia_genero.pdf

SEP. (2017). Programa Nacional de Convivencia Escolar. Documento base. Secretaría de Educación Pública. https://www.gob.mx/cms/uploads/attachment/file/195743/DB_PNCE_260217.pdf

Spinrad, T., \& Eisenberg, N. (2009). Empathy, prosocial behavior, and positive development in schools. In R. Gilman, E. S. Huebner, \& M. J. Furlong (Eds.), Handbook of positive psychology in schools (pp. 119-129). Routledge/Taylor \& Francis Group.

Sugai, J., \& Horner, R. (2006). A Promising Approach for Expanding and Sustaining School-Wide Positive Behavior Support. School Psychology Review, 35(2), 245-259. https://www.icareby.org/sites/www.icareby.org/files/spr352sugai.pdf

Tarragona, M. (2016). Historia de la victimización materna y su impacto sobre la salud mental de los mentores en entornos de violencia de pareja [Universidad Autónoma de Barcelona]. https://ddd.uab.cat/pub/tesis/2016/hdl_10803_367456/mjto1de1.pdf

Trucco, D., \& Inostroza, P. (2017). Las violencias en el espacio escolar. CEPAL / UNICEF. https://repositorio.cepal.org/bitstream/handle/11362/41068/4/S1700122_es.pdf 
UNESCO. (2015). La educación para todos 2000-2015. Logros y desafíos. Informe de seguimiento de la EPT en el mundo. (1st ed.). Organización de las Naciones Unidas para la Educación, la Ciencia y la Cultura. https://unesdoc.unesco.org/ark:/48223/pf0000232435_spa

UNESCO. (2019). Behind the numbers: Ending school violence and bullying. Organización de las Naciones Unidas para la Educación, la Ciencia y la Cultura. https://unesdoc.unesco.org/ark:/48223/pf0000366483

UNICEF. (2004). Desarrollo psicosocial de los niños y las niñas (2nd ed.). Fondo de las Naciones Unidas para la Infancia. https://www.politecnicometro.edu.co/biblioteca/primerainfancia/drrollo psicosocial unicef.pdf

UNICEF. (2014). Ocultos a plena luz. Un análisis estadístico de la violencia contra los niños. Resumen. https://www.unicef.org/ocultos-a-plena-luz.pdf

UNICEF. (2017). Violencia en las vidas de los niños y los adolescentes, una situación habitual. Datos fundamentales. https://www.unicef.org/publications/files/Violence_in_the_lives_of_children_Key_findin gs_Sp.pdf

UNICEF. (2018). Informe anual México 2018. https://unicef.org.mx/Informe2018/InformeAnual-2018.pdf

UNICEF. (2019a). Panorama estadístico de la violencia contra niñas, niños y adolescentes en México.

PanoramaEstadistico.pdf

https://www.unicef.org/mexico/media/1731/file/UNICEF

UNICEF. (2019b, June 10). Sueño con la paz. Colección de escritos y dibujos hechos por niños de la ex Yugoslavia. https://www.unicef.org/spanish/education/focus_peace_education.html

Varela, J., Tijmes, C., \& Sprague, J. (2009). Programa de prevención de la violencia escolar. Paz Educa. Fundación Paz Ciudadana. https://pazeduca.cl/wpcontent/uploads/2017/02/Programa-de-prevención-de-violencia-escolar-Paz-Educa.Implementación-y-resultados-versión-2005-2006.pdf

Villafuerte, D., \& García, M. (2014). Tres ciclos migratorios en Chiapas: interno, regional e internacional. Revista Migración y Desarrollo, 12(22), 3-37. https://doi.org/10.35533/myd.1222.dvs.mcga

Zárate, L., \& Cervantes, J. (2011). Violencia en niños de primaria. Revista Electrónica de Psicología Iztacala, $14(4)$, $1-13$. https://www.iztacala.unam.mx/carreras/psicologia/psiclin/vol14num4/Vol14No4Art1.pdf 